\title{
ESTRATÉGIAS REPRODUTIVAS DE SETE ESPÉCIES DE PEIXES DAS ÁGUAS COSTEIRAS DO RIO GRANDE DO NORTE, BRASIL
}

\author{
M. R. OLIVEIRA* , A. L. S. MORAIS, A. M. SILVA, J. T. A. X. LIMA, M. M. CARVALHO, N. T. CHELLAPPA e S. \\ CHELLAPPA \\ Departamento de Oceanografia e Limnologia, Centro de Biociências, Universidade Federal do Rio Grande do \\ Norte \\ monicaufrn@yahoo.com.br*
}

Artigo submetido em janeiro/2015 e aceito em outubro/2015

DOI: 10.15628/holos.2015.3601

\section{RESUMO}

O presente trabalho verificou as estratégias reprodutivas de sete espécies de peixes marinhos da região costeira do Rio Grande do Norte, Brasil. Traços da história de vida dos peixes em ambientes relativamente estáveis sugerem um modelo de três estratégias reprodutivas: (1) estrategistas oportunistas, são peixes de pequeno tamanho corporal com primeira maturação precoce, período de vida curto com baixa fecundidade; (2) estrategistas sazonais, são peixes de tamanho corporal intermediário, primeira maturação intermediária e fecundidade intermediária a alta; e (3) estrategistas de equilíbrio, são peixes de tamanho corporal grande, primeira maturação tardia com longo período de vida, além de alta fecundidade. Os aspectos reprodutivos de Hirundichythys affinis, Hemiramphus brasiliensis, Pomadasys corvinaeformis,
Oligoplites palometa, Scomberomorus brasiliensis, Lutjanus synagris e Mugil curema foram verificados, considerando-se o tamanho do corpo, proporção sexual, comprimento de primeira maturação sexual, aspectos do desenvolvimento das gônadas, fecundidade, tipo de desova e período reprodutivo dos peixes. Os resultados indicam que $H$. affinis, $H$. brasiliensis, $P$. corvinaeformis e $O$. palometa podem ser considerados como estrategistas oportunistas. Entretanto, S. brasiliensis, L. synagris e $M$. curema podem ser considerados como estrategistas de equilíbrio. Este estudo fornece informações sobre os aspectos reprodutivos dos peixes dos estoques pesqueiros das águas costeiras do Rio Grande do Norte, Brasil.

PALAVRAS-CHAVE: reprodução de peixes; águas costeiras; período de desova; peixes marinhos tropicais.

\section{REPRODUCTIVE STRATEGIES OF SEVEN FISH SPECIES FROM THE COASTAL WATERS OF RIO GRANDE DO NORTE, BRAZIL}

\begin{abstract}
This work determined the reproductive strategies of seven marine fish species from the coastal region of Rio Grande do Norte, Brazil. Life history traits of fish in relatively stable environments suggest a model of three reproductive strategies: (1) opportunistic strategists, fish with small body size, early first sexual maturity, short-life span and low fecundity; (2) seasonal strategists, fish with body size and first sexual maturity both intermediary, with intermediate to high fecundity; and (3) equilibrium strategists, fish of big body size, long life span, late first sexual maturity, with high fecundity. Aspects of reproductive strategies of Hirundichythys affinis, Hemiramphus brasiliensis, Pomadasys corvinaeformis,
\end{abstract}

Oligoplites palometa, Scomberomorus brasiliensis, Lutjanus synagris and Mugil curema were verified according to the body size, sex ratio, length at first sexual maturity, aspects of gonad development, fecundity, type of spawning and reproductive period of the fish. The results suggest that $H$. affinis, $H$. brasiliensis, $P$. corvinaeformis and $O$. palometa could be considered as opportunistic strategists. On the other hand $S$. brasiliensis, Lutjanus synagris and $M$. curema could be considered as equilibrium strategists. This study provides information on the reproductive aspects of fish which constitute the fishery stocks of the coastal waters of Rio Grande do Norte, Brazil.

KEYWORDS: Fish reproduction; coastal waters; spawning period; tropical marine fish. 


\section{INTRODUÇÃO}

A região Nordeste vem se destacando na produção de pescado marinho, sendo responsável pela maior proporção da produção nacional, com 186.012,0 t (MPA, 2012). A pesca marinha nesta região caracteriza-se pela predominância da pesca artesanal sobre a industrial (IBAMA, 2008). Este tipo de pesca apresenta elevada riqueza das espécies, que são consideradas recursos pesqueiros de importância econômica e ecológica (SILVA et al., 2012; CARVALHO et al., 2014). A região costeira do Estado do Rio Grande do Norte é constituída de vinte e cinco municípios costeiros, com 93 comunidades pesqueiras. Nestas comunidades, a pesca artesanal é responsável por cerca de $70 \%$ da produção total, a qual evidencia sua importância socioeconômica (OLIVEIRA et al., 2013).

O peixe-voador, Hirundichythys affinis (Günther, 1866), agulha-preta, Hemiramphus brasiliensis (Linnaeus, 1758), coró, Pomadasys corvinaeformis (Steindachner, 1868), tibiro, Oligoplites palometa (Cuvier,1832), serra, Scomberomorus brasiliensis Collette, Russo \& ZavalaCamin, 1978, ariacó, Lutjanus synagris (Linnaeus, 1758) e tainha, Mugil curema Valenciennes, 1836, são espécies de peixes marinhos que apresentam importante valor econômico e social na pesca artesanal do Estado do Rio Grande do Norte, Brasil. Essas espécies estão entre os mais importantes recursos pesqueiros distribuídos na região Nordeste do Brasil. A poluição dos ambientes marinhos costeiros e a pesca desordenada de recursos pesqueiros, tem causado uma redução de diversos recursos marinhos e estuarinos (VIEIRA; LIMA, 2003; OLIVEIRA, 2010; SILVA, et al., 2012). Desta forma, seus estoques estão sendo ameaçados pela pesca predatória, impactando as necessidades alimentares e econômicas dos pescadores artesanais, que retiram desses ecossistemas, parte de sua subsistência (DIAS et al., 2007; CHELLAPPA et al., 2010; CAVALCANTE et al., 2012; OLIVEIRA et al., 2012). Portanto, é considerado essencial realizar estudos que auxiliem na gestão desses recursos marinhos. Pesquisas sobre os aspectos reprodutivos de peixes têm servido de parâmetros para o entendimento dos mecanismos que envolvem a perpetuação das espécies e também para fornecer subsídios para a gestão pesqueira (MURUA et al., 2003; CAVALCANTE et al., 2012).

Os peixes apresentam diferentes estratégias reprodutivas para maximizar sua produção e garantir a sobrevivência dos descendentes até a idade adulta. Estas estratégias são aprimoradas por seleção natural às pressões fisiológicas e ambientais sobre a reprodução. Cada estratégia reprodutiva constitui um conjunto de táticas reprodutivas que a espécie manifesta para ter sucesso ao longo das gerações, de modo a garantir o equilíbrio da população (POTTS; WOOTTON, 1984; WINEMILLER; ROSE, 1992; MURUA; SABORIDO-REY, 2003; MORGAN 2004). Estudos sobre as táticas reprodutivas, tais como, tamanho corporal, a relação peso-comprimento, proporção sexual, o tamanho da primeira maturação sexual, o desenvolvimento das gônadas, a fecundidade, índice gonadosomático (IGS), tipo e época de desova dos peixes são informações importantes para tomada de medidas racionais para a regulamentação da pesca e conservação dos estoques pesqueiros (MORGAN 2004; LIMA et al. 2007; OLIVEIRA et al 2011). Existem três tipos de estratégias reprodutivas: (1) estrategistas oportunistas, são peixes de pequeno tamanho corporal com primeira maturação precoce, período de vida curto com baixa fecundidade (2) estrategistas sazonais, são peixes do tamanho corporal intermediário, primeira maturação intermediária e fecundidade intermediária a alta; e (3) estrategistas de equilíbrio, são peixes de tamanho corporal grande, primeira maturação tardia com longo período de vida, além de alta fecundidade (WINEMILLER; ROSE, 1992; KING; MCFARLANE, 2003). 
A relação peso-comprimento é uma importante ferramenta utilizada para determinação do peso quando apenas as medidas de comprimento estão disponíveis, que indica a condição dos peixes e permite comparações dos parâmetros entre espécies de diferentes regiões (OSCOZ et al., 2005). A proporção sexual constitui informação básica para avaliar o potencial reprodutivo e estimar o tamanho do estoque populacional (STRATOUDAKIS et al., 2006; CAVALCANTE et al., 2012). O comprimento médio em que os indivíduos alcançam a maturidade sexual é importante para determinar o tamanho mínimo permitido para captura, uma vez que os estoques pesqueiros estão comprometidos com a sobre exploração (KING, 1997; CHELLAPPA et al., 2010).

O presente trabalho verificou as táticas reprodutivas do peixe-voador, Hirundichythys affinis (Günther, 1866), agulha-preta, Hemiramphus brasiliensis (Linnaeus, 1758), coró, Pomadasys corvinaeformis (Steindachner, 1868), tibiro, Oligoplites palometa (Cuvier,1832), serra, Scomberomorus brasiliensis Collette, Russo \& Zavala-Camin, 1978, ariacó, Lutjanus synagris (Linnaeus, 1758) e tainha, Mugil curema Valenciennes, 1836, das águas costeiras do Rio Grande de Norte, Brasil. As táticas reprodutivas foram investigadas afim de determinar as estratégias reprodutivas de peixes marinhos que apresentam importante valor econômico e social na pesca artesanal do Estado do Rio Grande do Norte, Brasil.

\section{MÉTODOS E MATERIAIS}

\subsection{Local de estudo}

O Estado do Rio Grande do Norte possui uma extensão aproximadamente de $420 \mathrm{~km}$,

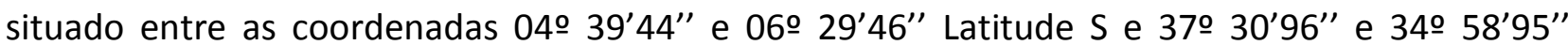
Longitude W. É caracterizado por praias arenosas que se estendem desde cidade de Tibau do Sul a Baía Formosa e são bastante exploradas pela população como fonte de renda através da pesca artesanal, lazer e recreação. Os peixes em consideração (Figura 1) foram capturados nas águas costeiras do Rio Grande Norte, na praia de Ponta negra (05o 52'47 30" Latitude S e 350 10'6

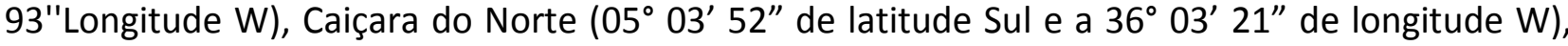
Praia da Redinha (05 45' 00" S e 35 10' 35" W) e vários outros locais nas águas costeiras do Estado do Rio Grande do Norte.

\subsection{Captura dos peixes}

Os exemplares de peixes foram capturados mensalmente durante o período de agosto de 2010 a julho de 2011 com o auxílio dos pescadores que utilizaram rede de fundo e rede de espera com barco a motor. Os espécimes capturados foram numerados, pesados, medidos e identificados utilizando as chaves de taxonomia. Foi verificada a proporção sexual, o comprimento total, o peso total, a relação peso-comprimento, tipo de crescimento, primeira maturação sexual, desenvolvimento das gônadas, fecundidade, tipo de desova e o período de desova.

As gônadas foram retiradas, pesadas (g), identificadas quanto ao sexo e o estádio de desenvolvimento gonadal (VAZZOLER, 1996). A relação peso-comprimento foi determinada pela equação $W=a L b$, onde $W$ é o peso total $(g)$, $L$ é o comprimento total $(\mathrm{cm})$, a é o interceptor (coeficiente de crescimento inicial ou o fator de condição) e o b é coeficiente alométrico (coeficiente de crescimento ou taxa de crescimento relativa de peixe) (LE CREN, 1951; JOBLING, 
2002). A proporção sexual foi dada como M:F calculada em relação ao número total de machos / número total de fêmeas (VAZZOLLER, 1996). A determinação da primeira maturação sexual foi feita pela distribuição da frequência relativa de machos e fêmeas adultas em classes de comprimento total para o período de estudo (MORENO et al., 2005).

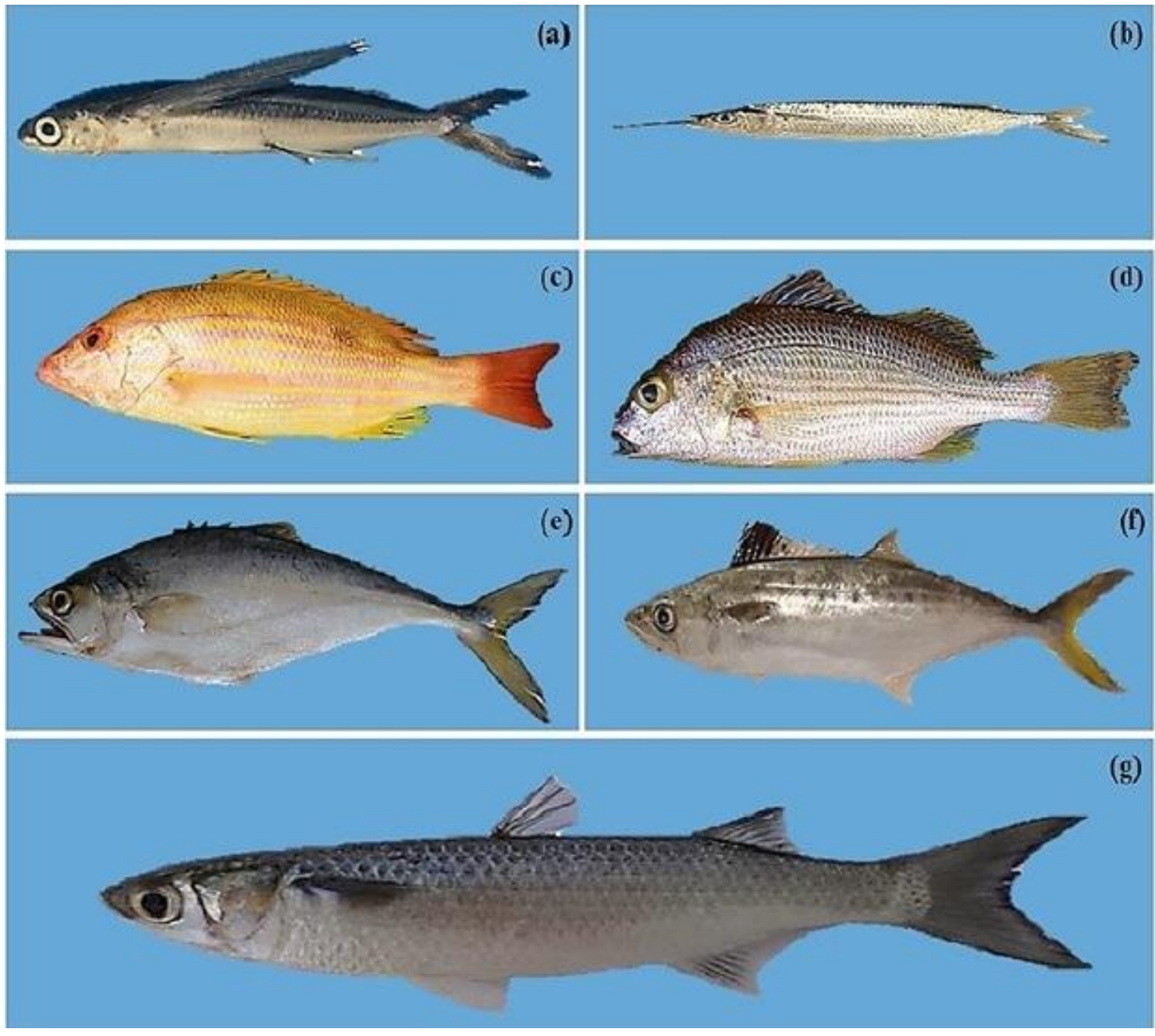

Figura 1: Espécies de peixes marinhos, capturadas no Litoral do Rio Grande do Norte: Hirundichythys affinis (a), Hemiramphus brasiliensis (b), Lutjanus synagris (c), Pomadasys corvinaeformis (d), Oligoplites palometa (e), Scomberomorus brasiliensis (f) e Mugil curema (g).

A classificação macroscópica do desenvolvimento gonadal foi descrita de acordo com Vazzoler (1996), sendo observado o tamanho da gônada em relação à cavidade celomática, coloração, presença de vasos sanguíneos, grau de turgidez das gônadas e aspecto dos ovócitos. Fecundidade absoluta foi avaliada através do número de ovócitos que uma fêmea irá desovar no período reprodutivo. O tipo de desova foi avaliado através da medição do diâmetro dos ovócitos (em $\mu \mathrm{m}$ ) e através da análise histológico do desenvolvimento dos ovócitos. O período reprodutivo foi determinado através da distribuição das frequências relativas (\%) de cada estádio de maturação das gônadas e a variação da média mensal do índice gonadossomático (IGS), considerando os sexos separados (VAZZOLER, 1996). 


\section{RESULTADOS E DISCUSSÃO}

\subsection{Peso e comprimento dos peixes}

A amplitude de comprimento total e o peso total das sete espécies de peixes marinhos em consideração estão apresentados na Tabela 1. O comprimento total dos machos do peixe-voador, $H$. affinis variou de 25,25 a $27,714 \mathrm{~cm}(26,546 \pm$ DP 1,408$)$ e o peso corporal dos machos da mesma espécie variou de 126,03 a 199,21 g $(150,24 \pm 31,23)$. Enquanto que o comprimento das fêmeas de $H$. affinis variou de 26,305 a $27,727 \mathrm{~cm}(27,045 \pm 1,228)$ e o peso corporal das fêmeas variou de 13,604 a 21,261 g (15,475 $\pm 3,094)$. El-Deir (1998), obteve resultados similares, onde as fêmeas de $H$. affinis apresentaram maiores amplitudes de comprimento zoológico $(20,5$ a $26,3 \mathrm{~cm})$ que os machos $(18,9$ a $24,3 \mathrm{~cm})$.

O comprimento total dos machos de agulha-preta, $H$. brasiliensis variou de 15,5 a $33,5 \mathrm{~cm}$ $(23,1 \pm 2,2)$, enquanto a variação do peso total foi de 14 a $196,1 \mathrm{~g}(67 \pm 26,9)$, e para as fêmeas o comprimento total variou de 19,5 a $33 \mathrm{~cm}(24,3 \pm 2,6)$ e o peso total variou de 33,4 a $203,4 \mathrm{~g}$ $(73 \pm 28,2)$. Resultados similares foram encontrados para a espécie de $H$. brasiliensis na Venezuela e no Sul da Flórida (McBRIDE; THURMAN, 2003; YELIPZA et al., 2011; OLIVEIRA et al., 2012).

O comprimento total dos machos de coró, $P$. corvinaeformis variou de 4,9 a $14,7 \mathrm{~cm}$, com média de 10,27 , enquanto a variação do peso total foi de 1,4 a 41,3 g com média de 15,95. Para as fêmeas o comprimento total variou de 5,0 a 18,0 cm com média de 11,8 e o peso total variou de 1,4 a 74,0 g com média de 26,16. Na Baía de Guaratuba, Paraná, Brasil, foi capturado indivíduos com 7,6 a $20 \mathrm{~cm}$ de comprimento total (CHAVES, 1998).

Para o peixe tibiro, O. palometa o comprimento total dos machos variou de 19,8 a $57,5 \mathrm{~cm}$ $(2,61 \pm 0,668)$ e o peso total variou de $51,6 \mathrm{~g}$ a $942,0 \mathrm{~g}(154,3 \pm 153,3)$. O comprimento total das fêmeas variou de 20,0 a $32,0 \mathrm{~cm}(2,45 \pm 0,285)$, e o peso total das fêmeas variou de $45,3 \mathrm{~g}$ a $243 \mathrm{~g}$ $(116,07 \pm 47,33)$. Os machos apresentaram maiores comprimentos e peso, possivelmente por ter um maior numero de captura de machos $(n=49)$ do que as fêmeas $(n=29)$.

O comprimento total das fêmeas de serra, S. brasiliensis amostradas variou de 9,3 a 80,5 $\mathrm{cm}$ e o peso total variou de 8,1 a $3385 \mathrm{~g}$. Os resultados foram similares para a espécie de $S$. brasiliensis capturada na Praia de Ponta Negra, Natal (LIMA et al., 2005).

O comprimento total dos machos de ariacó, L. synagris variou de 12,0 a $36,0 \mathrm{~cm}(23,6 \pm 7,1)$, e o peso total variou de 17,5 a $891,5 \mathrm{~g}(250,9 \pm 214,9)$. As fêmeas apresentaram comprimento total variando de 21,5 a $36,5 \mathrm{~cm}(28,7 \pm 5,08)$, e o peso total variou de 125,5 a $743,0 \mathrm{~g}(377,2 \pm 214,1)$. 0 comprimento total e peso total foram maiores para fêmeas de $L$. synagris. Foram observados resultados similares para $L$. synagris nas águas costeiras do Rio Grande do Norte, na qual as fêmeas apresentaram maior comprimento total e peso total (CAVALCANTE et al., 2012).

O comprimento total de tainha, M. curema variou de 15,6 a $34,5 \mathrm{~cm}(24,9 \pm 4,1)$ e o peso total variou de 35,4 a $382 \mathrm{~g}(160,5 \pm 82,3)$. As fêmeas foram mais representativas do que os machos durante o estudo, isso possivelmente sugere que este ecossistema oferece condições adequadas para o desenvolvimento e sobrevivência dos juvenis (QUIÑONEZ-VELÁZQUEZ; LÓPEZ-OLMOS, 2011). 
As espécies de $H$. affinis, $H$. brasiliensis, L. synagris, $P$. corvinaeformis, S. brasiliensis e $M$. curema apresentaram maior comprimento total e peso total para as fêmeas em relação aos machos. As fêmeas em geral são mais pesadas do que os machos, devido às suas gônadas que tendem a ter maior massa em relação aos testículos (MURUA et al., 2003; OLIVEIRA et al., 2012).

Tabela 1: Análise descritiva do comprimento total e peso total para machos e fêmeas de sete espécies de peixes marinhos de valor comercial das águas costeiras do Rio Grande de Norte, Brasil.

\begin{tabular}{cccccccc}
\hline \multirow{2}{*}{ Espécie } & \multicolumn{4}{c}{ Comprimento total $(\mathbf{c m})$} & \multicolumn{3}{c}{ Peso total (g) } \\
& Sexo & média \pm DP & mínimo & máximo & média \pm DP & mínimo & máximo \\
\hline \multirow{2}{*}{ H. affinis } & $\mathrm{M}$ & $26,546 \pm 1,408$ & 25,25 & 27,714 & $150,24 \mathrm{~g} \pm 31,23$ & 126,03 & 199,21 \\
& $\mathrm{~F}$ & $2,7045 \pm 1,228$ & 26,305 & 27,727 & $154,75 \mathrm{~g} \pm 30,94$ & 136,04 & 212,61 \\
\hline \multirow{2}{*}{ H. brasiliensis } & $\mathrm{M}$ & $23,1 \pm 2,3$ & 15,5 & 33,5 & $67 \pm 26,9$ & 14,0 & 196,1 \\
& $\mathrm{~F}$ & $24,3 \pm 2,6$ & 19,5 & 33,0 & $73 \pm 28,2$ & 33,4 & 203,4 \\
\hline \multirow{2}{*}{ L. synagris } & $\mathrm{M}$ & $23,6 \pm 7,1$ & 12 & 36 & $250,9 \pm 214,9$ & 17,5 & 891,5 \\
& $\mathrm{~F}$ & $28,7 \pm 5,08$ & 21,5 & 36,5 & $377,2 \pm 214,1$ & 125,5 & 743 \\
\hline \multirow{2}{*}{ P.corvinaeformis } & $\mathrm{M}$ & 10,27 & 4,9 & 14,7 & 15,95 & 1,4 & 41,3 \\
& $\mathrm{~F}$ & 11,8 & 5,0 & 18,0 & 26,16 & 1,4 & 74,0 \\
\hline \multirow{2}{*}{ O. palometa } & $\mathrm{M}$ & $2,61 \pm 0,668$ & 19,80 & 57,50 & $154,3 \pm 153,3$ & 51,6 & 942,0 \\
& $\mathrm{~F}$ & $2,45 \pm 0,285$ & 20,00 & 32,00 & $116,7 \pm 47,33$ & 45,3 & 243,0 \\
\hline S. brasiliensis & $\mathrm{F}$ & $28,92 \pm 15,34$ & 9,30 & 80,5 & $265,6 \pm 430,9$ & 8,1 & 3385 \\
\hline M. curema & $\mathrm{F}$ & $24,9 \pm 4,1$ & 15,6 & 34,5 & $160,5 \pm 82,3$ & 35,4 & 382 \\
\hline
\end{tabular}

\subsection{Relação peso-comprimento e o tipo de crescimento}

O conhecimento da relação peso-comprimento, aliado a outros aspectos quantitativos tais como, fator de condição, crescimento, recrutamento e mortalidade de peixe, fornece informações básicas para o estudo da biologia pesqueira, importantes para um manejo racional da pesca em um ambiente. A relação peso-comprimento de seis espécies de peixes foi estimada.

$O$ crescimento de machos e fêmeas do peixe-voador, $H$. affinis $(\theta=2,208 \mathrm{M} ; 2,985 \mathrm{~F})$ e do tibiro, O. palometa $(\theta=0,996 \mathrm{M} ; 0,913 \mathrm{~F})$ foi do tipo alométrico negativo, indicando um maior incremento em comprimento do que em peso (ARAÚJO et al., 2011). Para agulha-preta, $H$. brasiliensis e tainha, $M$. curema $(\theta=2,985)$ o crescimento foi do tipo isométrico, onde o incremento em peso acompanha o crescimento em comprimento (OLIVEIRA et al., 2011). O crescimento de ariacó, L. synagris $(\theta=3,3647 \mathrm{M} ; 3,3152 \mathrm{~F})$ e serra, $S$. brasiliensis foi do tipo alométrico positivo. Se $\theta$ for igual a 3 o crescimento é isométrico; se $\theta$ for maior que 3 , é alométrico positivo, e se for menor que 3 o crescimento é alométrico negativo. Se o crescimento é isométrico, o incremento em peso acompanha o crescimento em comprimento, se é alométrico negativo há um incremento em peso menor do que em comprimento; e se é alométrico positivo há um incremento em peso maior do que em comprimento (JOBLING, 2002).

\subsection{Proporção sexual}

A proporção sexual do peixe-voador, $H$. affinis foi de $1 \mathrm{M}: 1,4 \mathrm{~F}$, não deferindo significativamente da proporção esperada (1:1). As fêmeas do peixe-voador predominaram do que os machos nas águas costeiras do município de Caiçara do Norte, RN. A predominância de fêmeas pode estar associada ao aumento acentuado das gônadas durante este período, o que tornaria as fêmeas mais susceptíveis a captura (ARAUJO; CHELLAPPA, 2002). Para agulha-preta, H. brasiliensis 
a proporção sexual foi de 1M:1,1F sem diferença significativa da proporção esperada (1:1). Para coró, $P$. corvinaeformis as fêmeas predominaram na população com a proporção sexual de $1 \mathrm{M}: 2,1 \mathrm{~F}$ diferindo significativamente da proporção esperada. Para a mesma espécie na Baía de Guaratuba, Paraná a proporção sexual aproximou da esperada (1:1) (CHAVES; CORRÊA, 2000). A espécie $O$. palometa apresentou uma diferença significativa, ocorrendo maior número de machos em relação ao número de fêmeas $2 \mathrm{M}: 1 \mathrm{~F}$. As fêmeas de 0 . palometa desovam longe das águas costeiras (DUQUE-NIVIA et al., 1995), o que, possivelmente explica a ocorrência maior dos machos capturados na área de coleta. Para $S$. brasiliensis a proporção sexual obtida foi de $2 \mathrm{M}: 1 \mathrm{~F}$, os machos predominaram na população. A predominância dos machos na população de $S$. brasiliensis, também foi registrada do Ceará ao sul d Bahia, sendo $59 \%$ machos e 41 \% fêmeas (NOBRÉGA, 2002). A espécie ariacó, L. synagris apresentou uma proporção sexual de 4,15M:1F, assim os machos predominaram na população diferindo significativamente da proporção esperada 1:1. Na costa de Pernambuco para a espécie de L. synagris, a proporção sexual foi de 1:2,43 na classe de 19 a $20 \mathrm{~cm}$ de comprimento total entretanto na classe de menor comprimento os machos predominaram (SILVA JUNIOR, 2009). Para tainha, M. curema, a proporção sexual foi de 1M:1F não deferindo significativamente da proporção esperada (1,03:1). Para a mesma espécie estudada em uma baía tropical brasileira a proporção foi 1M:3,77 (ALBIERI, 2009). Para Mugilídeos os machos apresentam em maior número em tamanhos menores enquanto que fêmeas prevalecem em maiores tamanhos (VAZZOLLER, 1996; IBAÑEZ-AGUIRRE \& GALLARDO-CABELLO, 2004). As fêmeas predominaram para as espécies de $H$. affinis, $H$. brasiliensis e $P$. corvinaeformis e para as espécies de $L$. synagris, $O$. palometa e $S$. brasiliensis os machos predominaram.

Geralmente a proporção sexual ocorre de 1:1, mas ao longo do ciclo de vida dos peixes, a proporção sexual pode variar em função de diversos fatores que atuam de forma diferente sobre os indivíduos de cada sexo. A mortalidade e o crescimento são fatores que podem atuar de modo diferencial sobre machos e fêmeas, determinando o predomínio de indivíduos de um dos sexos (VAZZOLLER, 1996). A proporção sexual poderá ser afetada por diferentes fatores relacionados com a pesca, estações do ano, cardumes em áreas de alimentação e desova (LASIAK, 1982).

\subsection{Primeira maturação gonadal (L50)}

As fêmeas de $H$. affinis, maturam primeiras do que os machos. O tamanho no qual $50 \%$ dos machos e fêmeas iniciaram o processo de maturação gonadal foi de $23,8 \mathrm{~cm}$ e $23,0 \mathrm{~cm}$ de comprimento total, respectivamente, para a espécie de $H$. affinis capturado em Caiçara do Norte. O comprimento médio da primeira maturação de $H$. brasiliensis $\left(L_{50}\right)$ para os machos foi de $28 \mathrm{~cm}$ e das fêmeas foi de $20 \mathrm{~cm}$ de comprimento total. A primeira maturação sexual para $H$. brasiliensis, foi de 19,3 cm para fêmeas e 18,6 cm para os machos (MONTEIRO, 2003; GONDOLO, 2008). Para P.corvinaeformis o comprimento da maturação foi de $10,3 \mathrm{~cm}$ para os machos e $10,4 \mathrm{~cm}$ para as fêmeas. No Ceará para essa espécie foi obtido comprimento entre 4,0 e 18,0 cm para juvenis (COSTA et al., 1995). Para S. brasiliensis o comprimento da primeira maturação para machos foi $34,5 \mathrm{~cm}$ e $28 \mathrm{~cm}$ para fêmeas de comprimento total da espécie. Entre os anos de 1963-1986 a média da L50 foi 52 cm CT e a participação de juvenis foi de 8,1\% (GESTEIRA; MESQUITA, 1976); entre 1970-1975 o percentual foi de 14,2\% de imaturos capturados, com indivíduos da espécie atingindo até $120 \mathrm{~cm}$ CT (FONTELES-FILHO, 1988); entre 1998-2000 a média do comprimento decresceu para $38,2 \mathrm{~cm}$ e os indivíduos da costa do Nordeste brasileiro não atingiram medidas 
acima de $86 \mathrm{~cm}$ (LUCENA et al., 2001). O decréscimo do comprimento médio individual, estimado em 31,25 cm CT demonstra uma possível ocorrência de sobre pesca, o que causa uma redução no recrutamento da serra para o estoque capturável e no número potencial de indivíduos para iniciar o ciclo reprodutivo (LIMA et al., 2007). L. synagris apresentou para sexo agrupado um comprimento $\left(L_{50}\right)$ de $25,7 \mathrm{~cm}$. O $L_{50}$ para as fêmeas de $L$. synagris na costa de Pernambuco foi estabelecido em $20 \mathrm{~cm}$ de comprimento total, deferindo dos nossos resultados (SILVA JUNIOR, 2009). Em Bermudas nosso resultado foi similar ao encontrado para espécie de L. synagris, onde o tamanho de maturação $50 \%$ foi estimado em 24,5 e $23,5 \mathrm{~cm}$ de comprimento zoológico para fêmeas e machos, respectivamente (LUCKHURST et al., 2000).

O tamanho da primeira maturação gonadal para sexos agrupados de $M$. curema foi $24.3 \mathrm{~cm}$ de comprimento total corroborando com o estudo para a mesma espécie na Venezuela (MARÍN et al., 2003). Entretanto, o L50 para a mesma as fêmeas da mesma espécie foi $27.8 \mathrm{~cm}$ no Golfo do México (IBÁÑEZ-AGUIRRE AND GALLARDO-CABELLO, 2004).

As espécies de $H$. affinis, $H$. brasiliensis e $S$. brasiliensis mostraram que as fêmeas maturaram primeiro do que os machos. Para a administração racional dos estoques pesqueiros, é necessário conhecer o comprimento da primeira maturação gonadal, pois constitui um elemento para fixação dos tamanhos mínimos de captura e para a determinação do tamanho das malhas da rede (ARAÚJO; CHELLAPPA, 2002). Mudanças na quantidade de reservas de energia disponíveis para o desenvolvimento das gônadas, ou alterações no crescimento podem ser fatores que influenciem o comprimento da maturação (MORGAN, 2004; ENGELHARD; HEINO, 2004; CAVALCANTE et.al 2012; OLIVEIRA et al., 2012; SILVA et al., 2012). O conhecimento do tamanho da primeira maturação está entre as informações que contribuem na adoção de uma série de medidas de manejo que tem como objetivo a sustentabilidade da exploração pesqueira.

\subsection{Desenvolvimento gonadal}

A utilização de escalas macroscópicas de maturidade das gônadas contribui para o conhecimento biológico na descrição dos fenômenos do ciclo reprodutivo dos peixes e auxilia na compreensão do período reprodutivo da espécie. Contudo, a análise histológica é fundamental para uma melhor identificação dos estádios de desenvolvimento das gônadas. As características macroscópicas dos testículos dos machos de $H$. affinis apresentaram três estádios de maturação, imaturo, em maturação e maduro, para as fêmeas, foram cinco estádios de maturação, imaturo, em maturação I, em maturação II, maduro e esvaziado. Os resultados foram semelhantes com o trabalho realizado por Araújo e Chellappa (2002) que registraram quatro estádios de maturação para machos e fêmeas de $H$. affinis: Imaturo, em maturação I, em maturação Il e maduro. A espécie $H$. brasiliensis apresentou quatro estádios de maturação para machos e fêmeas, imaturo, em maturação, maduro e esvaziado. Os estudos macroscópicos das gônadas de $H$. brasiliensis indicaram que ambos os sexos apresentaram quatro estádios de desenvolvimento gonadal, sendo imaturo, em maturação, maduro e esvaziado. Os presentes resultados assemelham-se com os registrados para três espécies da família Hemiramphidae nas águas costeiras da Austrália, no qual as fêmeas apresentaram mudanças de tamanho e cor ao longo desenvolvimento ovariano (HUGHES; STEWART, 2006).

As características microscópicas dos exemplares de $P$. corvinaeformis permitiram estabelecer quatro estádios de desenvolvimento gonadal para os machos: imaturo, em maturação, 
maduro e esvaziado e sete estágios para as fêmeas: imaturo, em maturação inicial, em maturação final, maduro inicial, maduro intermediário, maduro final e esvaziado. Para ambos os sexos de $O$. palometa, a análise macroscópica permitiu a identificação de quatro estádios distintos de maturação: imaturo, em maturação, maduro e esvaziado. Os estádios de desenvolvimento macroscópicos das gônadas de $O$. palometa seguem os mesmo padrão de padrão de descrição dos peixes Carangideos (ARAÚJO, 2008). Os estádios macroscópicos dos ovários das fêmeas de $S$. brasiliensis, permitiu a visualização de quatro estágios: imaturo, em maturação, maduro e esvaziado. Estudos realizados no litoral ocidental do Maranhão, Brasil, com a espécie S. brasiliensis, foram similares ao nosso resultado, com quatro estádios de desenvolvimento gonadal (LIMA, 2004).

As características macroscópicas dos ovários e testículos de L. synagris revelaram quatro estádios de desenvolvimento: imaturo (I), em maturação (II), maduro (III) e esvaziado (IV). No estádio I, os ovários e testículos apresentaram-se filiformes e tamanhos reduzidos. Nos estádios II e III, os ovários mudaram a coloração de translúcida para avermelhada, aumentando de tamanho com presença de ovócitos visíveis. Os testículos apresentaram tamanhos variados de acordo com o grau de desenvolvimento, tornaram-se mais espessos com coloração esbranquiçada. No estádio IV, as gônadas mostraram tamanho reduzido com um aspecto flácido. Nossos resultados corroboram com os de Souza-Júnior et al. (2008) que registraram quatro estádios de maturação gonadal para a mesma espécie no Ceará. A descrição macroscópica dos ovários e testículos de $L$. synargis demonstra como ocorre o desenvolvimento gonadal no ciclo reprodutivo, fornecendo dados para indicar o período reprodutivo da espécie na região.

A caracterização macroscópica dos ovários de $M$. curema revelou quatro estádios de maturação ovariana assemelhando-se à descrista para a mesma espécie na Baía de Sepetiba (ALBIERI et al., 2009). No Caribe para M. curema observaram macroscopicamente seis estádios de desenvolvimento ovariano para M. curema, capturado no Caribe: repouso, em maturação inicial, em maturação final, maduro, parcialmente desovado e desovado (SOLOMON; RAMNARINE, 2007), A utilização de escalas macroscópicas contribui para o conhecimento biológico, mas a utilização de análises histológicas e medição de ovócitos diminuem a chance de incorrer erros (WEST, 1990).

\subsection{Fecundidade e tipo de desova}

A fecundidade absoluta de $H$. affinis variou de 7398 a 10021 (9092 \pm 1153.2 ) ovócitos vitelogênicos. Este estudo registrou uma média de 9.092 ovócitos vitelogênicos para $H$. affinis, embora um valor mais baixo foi registrado em um estudo anterior (ARAÚJO; CHELLAPPA, 2002). Os ovócitos de $H$. affinis apresentaram diâmetros variando de $100 \mu \mathrm{m}$ a $2500 \mu \mathrm{m}$, com um desenvolvimento sincrônico em dois grupos: um grupo do estoque de reserva com diâmetros dos ovócios inferiores a $1000 \mu \mathrm{m}$, e um grupo de ovócitos em desenvolvimento com diâmetros superiores a $1000 \mu \mathrm{m}$, indicando que a espécie apresenta a desova total, eliminando seus ovócitos maduros de uma só vez.

A fecundidade absoluta de $H$. brasiliensis variou entre 2.200 e 4.000 ovócitos vitelogênicos com uma média de 3.100 ovócitos maduros. Para a mesma espécie nas águas costeiras do sul da Flórida, foi registrada uma fecundidade de 1164 ovócitos para $100 \mathrm{~g}$ do peso da fêmea (McBRIDE; THURMAN, 2003; OLIVEIRA et al., 2012). O diâmetro dos ovócitos variou de $100 \mu \mathrm{m}$ a $800 \mu \mathrm{m}$. Os ovócitos foram classificados em dois grupos, um grupo do estoque de reserva com diâmetro menor 
que $300 \mu \mathrm{m}$, e um grupo de ovócitos em desenvolvimento com diâmetro maior que $600 \mu \mathrm{m}$. Dessa forma, a espécie $H$. brasiliensis, apresentou um desenvolvimento sincrônico dos ovócitos em dois grupos. A espécie tem desova total, eliminando seus ovócitos maduros de uma só vez (OLIVEIRA et al., 2012). A espécie $H$. brasiliensis possui baixa fecundidade quando comparada a outros peixes recifais e epipelágicos (MCBRIDE; THURMAN, 2003). Fazem pequenas desovas parceladas, principalmente durante o verão, mas podem ser encontrados indivíduos sexualmente maduros durante o ano todo (MCBRIDE et al.,1996; MCBRIDE; THURMAN, 2003; MONTEIRO, 2003).

A espécie $P$. corvinaeformis apresentou uma fecundidade absoluta variando entre 15.056 e 83.316 ovócitos. O diâmetro dos ovócitos de P. corvinaeformis variou de $110 \mu \mathrm{m}$ a $390 \mu \mathrm{m}$, foram classificados os ovócitos em dois grupos: um grupo de estoque de reserva, com diâmetro menor que $140 \mu \mathrm{m}$, e um grupo de ovócitos em desenvolvimento, com diâmetro maior que $141 \mu \mathrm{m}$. Os exemplares de $P$. corvinaeformis apresentaram desova total, eliminando seus ovócitos maduros de uma só vez.

S. brasiliensis apresentou uma fecundidade absoluta de 871.523 ovócitos e sua desova é total. Foram estimadas as fecundidades absoluta e relativa de $S$. brasiliensis em 2.047 .000 óvulos e 1.892 óvulos por grama do indivíduo, para o Estado do Ceará (GESTEIRA, 1972). A espécie $S$. brasiliensis possui gônadas com desenvolvimento de forma sincrônica, com dois grupos de ovócitos onde se encontra o estoque de reserva (ninhos germinativos e Fase II) com diâmetros inferiores a $120 \mu \mathrm{m}$, e outros ovócitos que iniciam na vitelogênese nas fases III, IV e V, até alcançar a fase VI com diâmetros entre 650 a $750 \mu \mathrm{m}$ de diâmetro. Estes são eliminados em desova total, verificada através da distribuição de frequência relativa do diâmetro dos ovócitos vitelogênicos (LIMA et al., 2007).

A fecundidade absoluta para M. curema foi de 245,828 ovócitos, e apresentou um desenvolvimento sincrônico dos ovócitos em dois grupos: um grupo de estoque de reserva, e um grupo de ovócitos em desenvolvimento, características de desova total. Para a mesma espécie na Baía de Sepetiba foi registrado a presença de dois grupos de ovócitos (ovócitos de estoque de reserva e pós-vitelogênicos) ao mesmo tempo em ovários maduros indicando um desenvolvimento sincrônico em dois grupos e a desova é total como também registrado no presente estudo (ALBIERI, 2009). Mugil curema um peixe que habita o mesmo local da espécie $P$. corvinaeformis apresentou uma fecundidade absoluta alta de 245,828 ovócitos (SILVA, 2003; OLIVEIRA et al., 2011; SILVA et al., 2012; OLIVEIRA et al., 2014).

\subsection{IGS e Período reprodutivo}

A espécie $H$. affinis, apresentou valores médios de IGS de 0,1 a 8,01 para machos e de 1,25 a 17,1para fêmeas. Verificou-se que seu período reprodutivo foi durante a época de chuvas, entre os meses de abril e junho. Os maiores valores de IGS são constatados no momento que antecede a desova, devido à hidratação dos óvulos. Este índice pode ser considerado como um dos indicadores do período de desova (ARAÚJO; CHELLAPPA, 2002; OLIVEIRA et al., 2015).

Para $H$. brasiliensis os valores médios mensais do IGS variaram de 0,18 a 0,39 para os machos e de 0,94 a 3,58 para as fêmeas. A espécie apresentou dois picos reprodutivos, de janeiro a março e entre maio e julho durante a época chuvosa. Para a mesma espécie no Sul da Flórida foi 
registrado um prolongado período de desova, que atingiu o pico no final da primavera e início do verão (McBRIDE et al., 2003).

A espécie $P$. corvinaeformis apresentou valores médios do IGS variando 0,012 a 1,150 para machos e de 0,012 a 5,490 para fêmeas. Foram observados dois picos de maiores valores nos meses de novembro e março, e a época de reprodução acontece entre outubro a junho. Para a mesma espécie no litoral norte de Santa Catarina a atividade reprodutiva foi muito intensa em duas estações do ano, primavera e verão (SOUZA; CHAVES, 2007).

Os valores de IGS da espécie 0 . palometa variaram de 0,07 a 0,15 para os machos enquanto que nas femeas essa variação foi de 0,07 a 1,64. No período de estiagem os machos apresentaram IGS médio de 0,13 (+0,03), enquanto que no período de chuva essa média foi de $0,11(+0,03)$. Não houve diferença significativa $(p>0,05)$ entre o IGS dos machos e os períodos chuvosos e de estiagem.

Para S. brasiliensis os valores de IGS variaram de 0,02 a 7,14 para as fêmeas. A atividade reprodutiva foi no período de março a junho e está relacionado com períodos chuvosos (CHELLAPPA et al., 2010).

Tabela 2. Táticas e Estratégias reprodutivos de Hirundichythys affinis; Hemiramphus brasiliensis; Pomadasys corvinaeformis; Oligoplites palometa; Scomberomorus brasiliensis; Lutjanus synagris e Mugil curema.

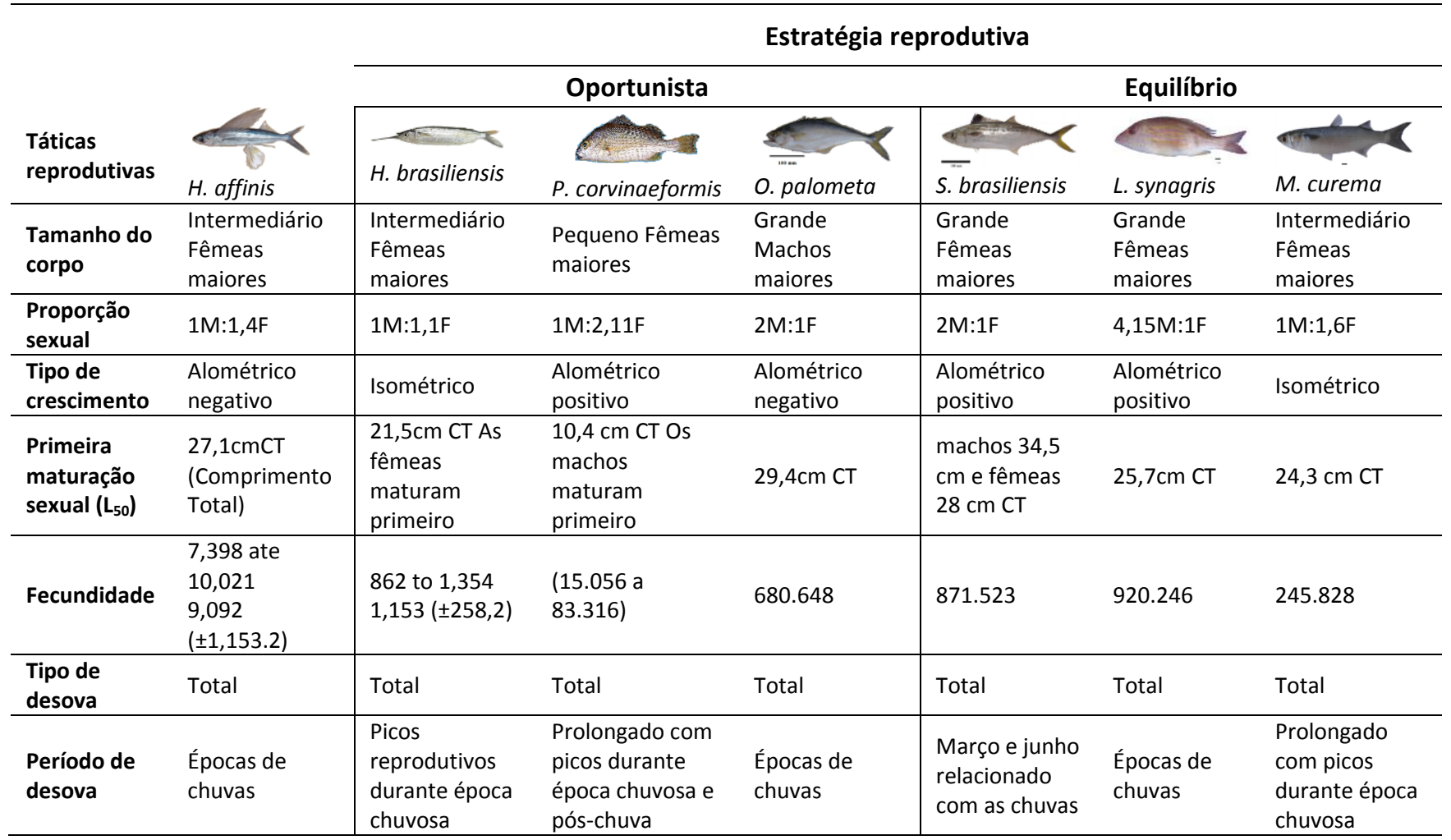

\section{CONCLUSÃO}

Os resultados fornecem informações sobre os aspectos reprodutivos das espécies de peixes marinhos que tem importância socioeconômica e ecológica para o Estado do Rio Grande do Norte. As informações como proporção sexual, relação peso-comprimento, tamanho mínimo de captura, desenvolvimento gonadal e fecundidade fornecem subsídios fornecer subsídios ao gerenciamento 
adequado do recurso, além de servir como base para novos estudos sobre a espécie. Os resultados indicam que $H$. affinis, $H$. brasiliensis, $P$. corvinaeformis e $O$. palometa são estrategistas oportunistas. S. brasiliensis, L. synagris e M. curema são estrategistas de equilíbrio.

\section{AGRADECIMENTOS}

Os autores agradecem ao Conselho Nacional de Desenvolvimento Científico Tecnológico (CNPq) e CAPES/MEC pela concessão de bolsas para realização da pesquisa. Os autores agradecem os pescadores artesanais que ajudaram a capturar peixes durante o período do estudo.

\section{REFERÊNCIAS BIBLIOGRÁFICAS}

1. ALBIERI, R.J. Biologia reprodutiva da tainha Mugil liza Valenciennes e do parati Mugil curema Valenciennes (Actinopterygii, Mugilidae) na Baia de Sepetiba, RJ, Brasil. Rio de Janeiro, 2009. Dissertação (Mestrado em Biologia Animal). Universidade Federal Rural do Rio de Janeiro. 63f, 2009.

2. ALBIERI, R.J., ARAÚJO, F.G., RIBEIRO, T.P. Gonadal development and spawning season of white mullet Mugil curema (Mugilidae) in a tropical bay. Journal of Applied Ichthyology, v.26, p.105109, 2009.

3. ARAÚJO, A. S., CHELLAPPA, S. Estratégia reprodutiva do peixe voador, Hirundichthys affinis Günther (Osteichthyes, Exocoetidae). Revista Brasileira de Zoologia, v.19, n.3, p. 691 - 703, 2002a.

4. ARAÚJO, G. S. Ecologia parasitária de isópodos e biologia reprodutiva em Tibiro, Oligoplites spp (Osteichthyes:Carangidae) das águas costeiras de Natal, Rio Grande do Norte. Natal, 2008. Dissertação de Mestrado em Ciências Biológicas, Universidade Federal do Rio Grande do Norte. 97f, 2008.

5. ARAújo, A. S., Oliveira, M. R., CAMPOS, C. E. C., YAMAMOTO, M. E., Chellappa, S. Características morfométricas-merísticas, peso-comprimento e maturação gonadal do peixe voador, Hirundichythys affinis (Günther, 1866). Revista Biota Amazônia, v. 1, n. 2, p. 33-40, 2011.

6. CARVALHO, M.M., MORAIS, A.L.S., GURGEL, T.A.B., OLIVEIRA, M.R., CHELLAPPA, S. Frequência de ocorrência e características morfológicas externos de peixes marinhos de Caiçara do Norte, Rio Grande do Norte, Brasil. Biota Amazônia. v. 4, n. 2, p. 55-63, 2014.

7. CAVAlCANTE, L. F. M., OliVeiRA, M. R., CHELLAPPA, S. Aspectos reprodutivos do ariacó, Lutjanus synagris nas águas costeiras do Rio Grande do Norte. Biota Amazônia, v. 2, n. 1, p. 45-50, 2012.

8. COSTA, P. S. R., SANTOS, M.A.M., ESPÍNOLA M.F.A., MONTEIRO-NETO. J. Biologia e biometria do coró, Pomadasys corvinaeformis (Steindachner) (Teleostei: Pomadasyidae), em Fortaleza, Ceará, Brasil. Arquivos de Ciências do Mar, v.29, n. 1-2, p. 20-27, 1995.

9. CHAVES, P. T .C. Estrutura populacional de Pomadasys corvinaeformis (Steindachner) (Teleostei, Haemulidae) na Baía de Guaratuba, Paraná, Brasil. Revista Brasileira de Zoologia, v.15, n.1, p. 203 - 209, 1998. 
10. CHAVES, P.T.C.; CORRÊA, C.E. Temporary use of a coastal ecosystem by the fish, Pomadasys corvinaeformis (Perciformes: Haemulidae), at Guaratuba Bay, Brazil. Revista Brasileira de Oceanografia, v. 48, n.1, p. 1-7, 2000.

11. CHELLAPPA, S., LIMA, J.T.A.X., ARAÚJO, A., CHELLAPPA, N.T. Ovarian development and spawning of Serra Spanish mackerel in coastal waters of Northeastern Brazil. Brazilian Journal of Biology, v.70, n.2, p. 631-637, 2010.

12. DIAS, T.L.P., ROSA, R.S., DAMASCENO, L.C.P. Aspectos socioeconômicos, percepção ambiental e perspectivas das mulheres marisqueiras da Reserva de Desenvolvimento Sustentável Ponta do Tubarão (Rio Grande do Norte, Brasil). Gaia Scientia, v.1, n.1, p. 25-35, 2007.

13. DUQUE-NIVIA, G.; ARTHURO, A. P.; SANTOS-MARTINEZ, A. Aspectos reproductivos de Oligoplites saurus y O. palometa (PISCES: CARANGIDAE) en La Ciénaga Grande de Santa Marta, Caribe Colombiam. Caribean Journal of Science, v.31 n. 3-4, p. 317-326, 1995.

14. EL-DEIR, A. C. A. Reprodução e Caracterização Morfométrica e Merística do Peixe-voador Hirundichthys affinis (Günther,1866) em Caiçara-RN. Recife, 1998. Dissertação de Mestrado, Universidade Federal Rural de Pernambuco, 92p, 1998.

15. ENGELHARD, G. H.; HEINO, M. Maturity changes in Norwegian spring-spawning herring before, during, and after a major population collapse. Fisheries Research, v. 66, p. 299-310, 2004.

16. FONTELES-FILHO, A.A. Sinopse de informações sobre a cavala, Scomberomorus cavalla (Cuvier) e a serra, Scomberomorus brasiliensis Collette, Russo \& Zavala-Camin (Pisces:Scombridae), no Estado do Ceará, Brasil. Arquivos Ciência do Mar, Fortaleza, v.27, p.21-48, 1988.

17. GESTEIRA, T.C.V. Sobre a reprodução e fecundidade da serra, Scomberomorus maculatus (Mitchill), no Estado do Ceará. Arquivos Ciência do Mar, Fortaleza, v.12, n.2, p.117-22, 1972.

18. GESTEIRA, T.C.V., MESQUITA, A.L.L. Época de reprodução, tamanho e idade na primeira desova da cavala e da serra, na costa do Estado do Ceará (Brasil). Arquivos Ciência do Mar, Fortaleza, v.16, n.2, p.83-86, 1976.

19. GONDOLO, G. F. Idade e crescimento de Hemiramphus brasiliensis (Linnaeus, 1758) no litoral de Pernambuco. Recife, 2008. Dissertação de Mestrado em Biologia animal, Universidade Federal de Pernambuco, 48f, 2008.

20. HUGHES, J.M.; STEWART, J. Reproductive biology of three commercially important Hemiramphid species in south-eastern Australia. Environmental Biology of Fishes, v. 75, p. 237-256, 2006.

21. IBAMA (Instituto Brasileiro do Meio Ambiente e dos Recursos Naturais Renováveis). Monitoramento da atividade pesqueira no litoral nordestino - Projeto ESTATPESCA- 2006. Tamandaré, PE. 384f, 2008.

22. IBAÑEZ-AGUIRRE, A.L., GALLARDO-CABELLO, M. Reproduction of Mugil cephalus and $M$. curema from a coastal lagoon to the northwest of the Gulf of Mexico. Bulletin Mar. Science, v.75, p.37-49, 2004.

23. JOBLING, M. Environmental factors and rates of development and growth. In HART, PJ. and REYNOLDS, JD. Handbook of fish biology and fisheries vol. 1, Fish Biology. Oxford: Blackwell Publishing Ltd. p. 97-122, 2002.

24. KING, M.G. Fisheries biology, assesment and management. Osney Mead, Oxford, England: 
Fishing news books, p.341, 1997.

25. KING, JR., McFARLANE, G.A. Marine fish life history strategies: applications to fishery management. Fisheries Management and Ecology, v. 10, p. 249-264. 2003.

26. LASIAK, A. Aspects of the reproductive biology of the Southern mullet Liza richardsoni from Algoa Bay, South Africa. A South African Zoology, v. 18, p. 89- 95, 1982.

27. LE CREN, E. D. The length-weight relationship and seasonal cycle in gonad weight I and condition in the perch (Perca fluviatilis). Journal of Animal Ecology. 20:201-19, 1951.

28. LIMA, J.T.A.X. Biologia reprodutiva e parasitismo por isópodes do serra, Scomberomorus brasiliensis (Collette, Russso \& Zavala-Camin, 1978) (Osteichthyes: Scombridae) no litoral do Rio Grande do Norte. Natal, 2004. Dissertação de Mestrado, Universidade Federal do Rio Grande do Norte, RN. 153f. 2004.

29. LIMA, J.T.A.X., FONTELES-FILHO, A.A., CHELLAPPA, S. Biologia reprodutiva da Serra, Scomberomorus brasiliensis (Osteichthyes: Scombridae), em águas costeiras do Rio Grande do Norte. Arquivos de Ciências do Mar, vol. 40, n. 1, p. 24-30, 2007.

30. LUCENA, F., LESSA, R., NÓBREGA, M. Presente status do estoque da serra Scomberomorus brasiliensis no Nordeste do Brasil. Anais do XII Congresso Brasileiro de Engenharia de Pesca, Foz do Iguaçu, 2001.

31. LUCKHURST, B. E., DEAN, J. M., REICHERT, M. Age, growth and reproduction of the lane snapper Lutjanus synagris (Pisces: Lutjanidae) at Bermuda. Marine Ecology Progress Series, v. 203, p. 255-261, 2000.

32. MARÍN, B. J., QUINTERO, A., BUSSIÈRE, D., DODSON, J. J. Reproduction and recruitment of white mullet (Mugil curema) to a tropical lagoon (Margarita Island, Venezuela) as revealed by otolith microstructure. Fishery Bulletin, v.101, p.809-821, 2003.

33. McBRIDE, RS., FOUSHEE, L., Mahmoudi, B. Florida's halfbeak, Hemiramphus spp., bait fishery. Marine Fishery Reviews, v. 58, p. 29-38, 1996.

34. McBRIDE, R. S., THURMAN, P. E. Reproductive biology of Hemiramphus brasiliensis and $H$. balao (Hemiramphidae): Maturation, Spawning Frequency, and Fecundity. Biological Bulletin, v. 204, p. 57-67, 2003.

35. MONTEIRO, A. Biologie et pêche des Aiguilles Hemiramphus brasiliensis (Linnaeus, 1758) et Hyporhamphus unifasciatus (Ranzani, 1842) (Poissins - Téléosteens - Hemiramphidae) dans la région Nord-Est du Brésil. Tese de doutorado apresentado pela Universite de Bretagne Occidentale, 210f, 2003.

36. MORENO, T., CASTRO, J.J., SOCORRO, J. Reproductive biology of the sand smelt Atherina presbyter Cuvier, 1829 (Pisces:Atherinidae) in the central-east Atlantic. Fisheries Research, v. 72, p. 121-131, 2005.

37. MORGAN, M. J. The relationship between fish condition and the probability of being mature in American plaice (Hippoglossoides platessoides). ICES Journal of Marine Science, v. 61, p. 6470, 2004.

38. MPA(Ministério da Pesca e Aquicultura). Boletim Estatístico da Pesca e Aquicultura/Brasil 2010. Brasília. 129p, 2012. 
39. MURUA, H., SABORIDO-REY, F. Female reproductive strategies of marine fish species of the North Atlantic. Journal of Northwest Atlantic Fishery Science, v. 33, p. 23-31, 2003.

40. MURUA, H., KRAUS, G., SABORIDO-REY, F., WITTHAMES, P.R, THORSEN A., JUNQUERA, S. Procedures to estimate fecundity of marine fish species in relation to their reproductive strategy. Journal of Northwest Atlantic Fishery Science. 33, p. 33-54, 2003.

41. NOBRÉGA, M. F. Idade, crescimento e avaliação de estoque da serra Scombereromus brasiliensis (Teleostei: Scombridae), na plataforma continental do Nordeste do Brasil. Recife, 2002. Dissertação de Mestrado em Biologia animal, Universidade Federal de Pernambuco, 2002.

42. OLIVEIRA, M.R. Biologia reprodutiva da tainha, Mugil curema Valenciennes, 1836 (Osteichthyes: Mugilidae) nas águas costeiras do Rio Grande do Norte. Natal, 2010. Dissertação de Mestrado em Ciências Biológicas, Universidade Federal do Rio Grande do Norte, 74f, 2010.

43. OLIVEIRA, M. R., COSTA, E.F.S., CHELLAPPA, S. Ovarian development and reproductive period of white mullet, Mugil curema in the coastal waters of Northeastern Brazil. Animal Biology Journal, v. 2, n. 4, p. 22-237, 2011.

44. OLIVEIRA, I. M. B; OLIVEIRA, M.R; YAMAMOTO, M. E; CHELLAPPA, S. Biologia reprodutiva de agulha-preta, Hemiramphus brasiliensis (Linnaeus, 1758) (Osteichthyes: Hemiramphidae) das águas costeiras do Rio Grande do Norte, Brasil. Biota Amazônia, v. 2, n. 2, p. 44-53, 2012.

45. OLIVEIRA, M. R; COSTA, E. F. S; ARAUJO, A. S; PESSOA, E. K. R; CARVALHO, M. M; CAVALCANTE, L. F. M; CHELLAPPA, S. Sex ratio and length-weight relationship for five marine fish species from Brazil. Journal of Marine Biology \& Oceanography, 1:2, 2012.

46. OlIVEIRA, M.R.; CARVALHO, M. M.; Souza, A.L.; MOLINA, W. F. YAMAMOTO, M. E.; CHELLAPPA, S. Caracterização da produção do peixe-voador, Hirundichthys affinis em Caiçara do Norte, Rio Grande do Norte, Brasil: durante 1993 a 2010. Biota Amazônia, v. 3, p. 23-32, 2013.

47. OLIVEIRA, M.R.; COSTA, E.F.S.; CHELLAPPA, S. Ovarian development and reproductive period of white mullet, Mugil curema in the coastal waters of northeastern Brazil. In: José Rosa Gomes; Sathyabama Chellappa. (Org.). Biology of Semiarid Tropical Fish. 1ed.New York, USA: Nova Scientific Publishers, v. 1, p. 21-34, 2014.OLIVEIRA, M. R., CARVALHO, M.M., SILVA, N.B., YAMAMOTO, M. E., CHELLAPPA, S. Reproductive aspects of the flyingfish, Hirundichthys affinis from the Northeastern coastal waters of Brazil. Brazilian Journal of Biology. v. 75, no. 1, p. 198 - 207, 2015.OSCOZ, J., CAMPOS, F., ESCALA, M.C. Weight length relationships of some fish species of the Iberian Penisula. Journal Applied Ichthyology, v. 21, p.73-74, 2005.

48. POTTS, GW., WOOTTON, RJ. Fish reproduction. London: Academic Press. 1984.

49. QUIÑONEZ-VELÁZQUEZ, C., LÓPEZ-OLMOS, J.R. Juvenile growth of white mullet Mugil curema (Teleostei: Mugilidae) in a coastal lagoon southwest of the Gulf of California. Latin American Journal of Aquatic Research, v.39, n.1, p.25-32, 2011.

50. SILVA, A.M. Aspectos reprodutivos do coró, Pomadasys corvinaeformis (Steindachner, 1868) (Osteichthyes: Haemulidae) das águas costeiras de Ponta Negra, Rio Grande do Norte. Natal, 2003. Dissertação de Mestrado em Ecologia, Universidade Federal do Rio Grande do Norte. 89f, 2003. 
51. SILVA JUNIOR, L. A. Pesca com covo e reprodução do ariocó Lutjanus synagris (Perciformes:Lutjanidae) na Costa de Pernambuco. Recife, 2009. Dissertação de Mestrado em Oceanografia, Universidade Federal de Pernambuco. 55f, 2009.

52. SILVA, A. M, OLIVEIRA, M. R, CHELLAPPA, S. Biologia reprodutiva do coró, Pomadasys corvinaeformis Steindachner (Osteichthyes: Haemulidae) das águas costeiras do Rio Grande do Norte, Brasil. Biota Amazônia, v. 2, n. 2, p. 15-24, 2012.

53. SOLOMON, F.N., RAMNARINE, I.W. Reproductive biology of white mullet, Mugil curema (Valenciennes) in the Southern Caribbean. Fisheries Research, v.88, p.133-138, 2007.

54. SOUZA, L.M., CHAVES, P.T. Atividade reprodutiva de peixes (Teleostei) e o defeso da pesca de arrasto no litoral norte de Santa Catarina, Brasil. Revista Brasileira de Zoologia, v. 24, n.4. p.1113-1121, 2007.

55. SOUSA JÚNIOR, V. B. de; SILVA, J. R. F.; SALLES, R. Análise ovariana do ariacó, Lutjanus synagris (Actinopterygii: Lutjanidae), e considerações sobre sua reprodução no estado do Ceará. Arquivos de Ciências do Mar, Fortaleza, v, 41, n. 1, p. 90-97, 2008.

56. STRATOUDAKIS, Y., BERNAL, M., GANIAS, K., URIARTE, A. The daily egg production method: recent advances, current applications and future challenges. Fish and Fisheries, v. 7, p.35-57, 2006.

57. VAZZOLER, AEAM. Biologia da Reprodução de Peixes Teleósteos: Teoria e Prática. Maringá: EDUEM. 169p, 1996.

58. VIEIRA, E.M.M.; LIMA, I.M.M.R. Um novo olhar para a extensão pesqueira: gênero na prática organizativa das mulheres marisqueiras. Prorenda Rural, PE. Extensão Pesqueira: Desafios Contemporâneos. Edições Bagaço: Recife, p.137-152, 2003.

59. WEST, G. Methods of assessing ovarian development in fishes: a Review. Australian Journal of Marine and Freshwater Research, v.41, p.199-222, 1990.

60. WINEMILLER, K.O., ROSE, K.A. Patterns of life-history diversification in North American fishes: implications for population regulation. Canadian Journal of Fisheries and Aquatic Sciences, v. 49, n.10, p. 2196-2218, 1992.

61. YELIPZA, L. R., ACOSTA, V., PARRA, B., LISTA, M. Aspectos biométricos de Hemiramphus brasiliensis (Peces:Hemirhamphidae), Isla de Cubagua, Venezuela. Zootecnia Tropical, v. 29, n.4, p. 385-398, 2011. 